\title{
Vodder manual lymphatic drainage technique versus Casley-Smith manual lymphatic drainage technique for cellulite after thigh liposuction
}

\author{
Esraa H. Rostom ${ }^{1,2}$, Amr B. Salama ${ }^{1,3}$ \\ ${ }^{1}$ Department of Physical Therapy for Surgery, Faculty of Physical Therapy, Cairo University, Cairo, Egypt \\ 2Department of Physical Therapy, Faculty of Medical Rehabilitation Sciences, Taibah University, El-Madinah El-Monawra, KSA \\ ${ }^{3}$ Department of Medical Rehabilitation Sciences, College of Applied Medical Sciences, Najran University, Najran, KSA \\ Adv Dermatol Allergol 2022; XXXIX (2): 362-367 \\ DOI: https://doi.org/10.5114/ada.2021.106042
}

\begin{abstract}
Introduction: Cellulite is one of the complications post liposuction. Cellulite causes changes in the lymphatic system. Manual lymphatic drainage is utilized as an effective treatment for enhancing cellulite.

Aim: To compare between Vodder Manual Lymphatic Drainage (MLD) Technique and Casley-Smith MLD Technique for cellulite after liposuction.

Material and methods: Thirty female patients with cellulite grade 3 after thigh liposuction participated in the study, and they were randomly divided into two equal groups: Group (A) that received Vodder MLD Technique and Bandage and Group (B) that received Casley-Smith MLD Technique and Bandage. The duration of the intervention was 8 weeks per participant, and each participant received 3 sessions per week.

Results: The results revealed that there was a significant improvement in both groups by using two different methods of treatment $(p<0.001)$, but there was no significant difference between the two study groups $(p>0.05)$. Conclusions: Both Vodder technique and Casley-Smith technique are effective in treatment of cellulite after thigh liposuction but there is no difference between them, hence any technique of MLD is recommended to achieve better improvement in this case.
\end{abstract}

Key words: Vodder Manual lymphatic drainage, Casley-Smith manual lymphatic drainage, cellulite, thigh liposuction.

\section{Introduction}

Liposuction is a common beauty approach and effective procedure of contour shaping. Liposuction is used to correct superficial and deep fat deposition. It has great ability for its usage in reconstructive and ablative surgical treatment [1].

Cellulite is also referred to as cottage cheese-like dimpling or "orange peel" of the pores and skin. It is widely seen as an aesthetically unacceptable hassle. Cellulite is manifested by topographic problems of subdermal tissue which include oedema or nodules. Its pathogenesis is complex and vague [2].

Cellulite causes changes in the lymphatic system that lead to an accumulation of fluids in subdermal tissue, especially within the cellular interstice. Those changes may be encouraged by way of hormones or by other factors that precipitate the aggregation of fluids and different substances in the cell interstice [3]. Liposuction actually worsens the dimpled skin appearance. Thus, Van Vliet and Avram do not recommend liposuction as a cellulite treatment [4].

Cellulite Severity Scale (CSS) was used for assessment of the degree of cellulite, a scale for the target investigation of cellulite grades. This scale morphologically measures the parts of cellulite, comparable to the quantity of obviously depressed lesions, the extent of the depressed lesions, the appearance of accelerated lesions, buckling, also with the previous traditional classification [5].

Nowadays, a wide assortment of cellulite treatment alternatives are available, ranging from topically-applied products to phototherapy. Currently, manual lymphatic drainage (MLD) is utilized as an effective treatment for enhancing cellulite with a stamped decrease within oedema, connective tissue and tissue thickness. Maximum helpful processes concentrate on the attractiveness of

Address for correspondence: Amr B. Salama, Department of Physical Therapy for Surgery, Faculty of Physical Therapy, Cairo University, Cairo, Egypt, phone: +966545427227, e-mail: amrpt81@yahoo.com Received: 22.01.2021, accepted: 2.03.2021. 
lipolysis. But, the effectiveness of such cellulite remedies seems to be lower than totally satisfactory [6].

The lymphatic system simulation strategy is a modality for cellulite treatment, which includes incitement of the physiological kind of frame, and hence the body spontaneously re-establishes its balance. This approach is effective in the management of cellulite [7].

Manual lymphatic drainage may be a hands-on remedy that is normally utilized as a part of complex decongestive therapy (CDT). CDT is comprised of MLD, compression dressing, lymph-lowering exercises, pores and skin care. MLD is comfortable and nicely persevered, well tolerated and may offer additional benefit to compression bandaging for swelling reduction. MLD may be a light but exceptionally particular hands-on treatment outlined to diminish lymph swelling by improving lymphatic drainage [8].

There are some schools for manual lymphatic drainage (Vodder, Casley-Smith, Leduc, Foldi), but they all agree on the fundamental aspects. The MLD should be performed by a well-trained therapist. MLD requires a long time of application (from 40 min to $1 \mathrm{~h}$ ) [9].

To date, no clinical research has directly compared the clinical efficacy of Vodder MLD Technique and Casley-Smith MLD Technique in the management of cellulite after liposuction. We believe that both interventions would be helpful in the treatment of cellulite after liposuction and that the direct comparison of the efficacy of both methods would provide insight into the potential decision-making processes for clinicians and patients regarding alternative therapies with medication or invasive treatments.

\section{Aim}

Therefore, this randomized clinical trial involves a comparative study between the efficiency of Vodder MLD technique and Casley-Smith MLD technique in the management of cellulite.

\section{Material and methods \\ Design}

This is a randomized control trial performed between September 2019 and October 2020 on participants with cellulite grade 3 after thigh liposuction, recruited from the Department of Surgery, Cairo University hospital. The study protocol was approved by the Faculty of Physical Therapy ethical committee. All patients signed written informed consent.

The participants were randomly assigned to one of two treatment groups: Group (A) received Vodder MLD technique and Bandage and Group (B) received CasleySmith MLD technique and bandage. The randomization process was performed using a sealed envelope method. The therapist prepared 30 sealed envelopes, each con- tained a card labelled either group A or B. Each participant has been asked to select an envelope that stated whether the patient was allocated to group A or B.

\section{Subjects}

Thirty female patients with cellulite grade 3 after thigh liposuction, after 1 month from surgery, aged between 30 and 45 years, undergoing physical therapy participated in the study. They were free from any pathological conditions like limb infection, malignancy, were not taking anti-coagulants, and deep vein thrombosis that might affect the results. Also, patients with skin diseases were excluded from the study.

\section{Material and measurement tools}

\section{Vodder MLD technique}

The objective of Vodder MLD Strategy is to extend the viability of the lymphatic pumps. This is done by using high pressure (a process known as pressure stage that includes manual pushing) and zero pressure (a process known as relaxation stage that includes manual contact). The therapist will employ his hands to move the skin within the heading of lymphatic flow, either by pushing or stretching the skin. Physical specialists utilize light pressure without causing redness. After the pressure stage, the therapist begins a relaxation stage. Amid this stage, the therapist keeps up manual contact with the skin but does not apply any pressure. The Vodder strategy utilizes four essential strokes: stationary circles, pumps, rotary, and scoop in different designs [10].

The affected limb was per skin and the therapist applied very soft pressure by hand motion on the skin and the subcutaneous tissue of the patient in the form of stationary circles, pumps and strokes. The applied pressure was divided between the lymph nodes, anastomosis, abdominal and the affected limb for $40 \mathrm{~min}$. The proximal part of the limb is continually cleared first, after that the drainage is extended peripherally and the carried-out pressure became for every area from distal to proximal.

\section{Casley-Smith MLD technique}

Casley-Smith MLD Strategy's main concept involves purging the trunk to begin to deliver the lymph from the peripheral area to other areas. The most effective is the limb massage. The proximal part of the limb is continuously cleared initially; at that point the massage is expanded distally. Beginning at the distal part and endeavouring to thrust the lymph into the un-empty area. Proximal areas are contraindicated. Other deeper trunk drainage can be applied by the therapist to help in the drainage of this area and create a bigger save for clearance from the thoracic area [11].

The affected limb and the trunk were per skin and the therapist applied soft massage on the truncal regions and the lymphotomies beside the affected part first for 
30 min to give the lymph from the periphery somewhere to go. Then the limb is massaged for 10 min starting at the distal area trying to move the lymph into the nonevacuated, while proximal areas were avoided.

\section{Bandage}

A layer of cotton (Velband) is applied firstly and then followed by protection at the points of support. Bandage of low elasticity (Comprilan) of various widths (using $6 \mathrm{~cm}$ for the toes and $8 \mathrm{~cm}$ for the remaining parts of the limb).

\section{Procedure}

Participants who met the inclusion criteria were randomly assigned into 2 groups, after initial evaluation, participants began the treatment on the same day. Participants received 3 sessions per week for 8 weeks. Before starting the treatment, all patients received full explanation regarding the purpose of the treatment, the therapeutic and physiological benefits of this method of treatment. All the measurements of each patient were taken for the comparison and each patient was placed in a comfortable position to clearly observe the affected area.

Group (A) $(n=15)$ received Vodder MLD Technique and Bandage in the following steps. The affected limb was per skin and the therapist applied very soft pressure with hand motion on the trunk for 30 min including 10 min for abdominal lymphatic drainage. Bandaging is applied at the end of each session. Bandages were wrapped with high pressure gradient at the most distal part of the limb which gradually decreases proximally. A layer of cotton (Velband) is applied firstly and then followed by protection at the points of support. A bandage of low elasticity (Comprilan) of various widths (using 6 $\mathrm{cm}$ for the toes and $8 \mathrm{~cm}$ for the remaining of the limb).

Group (B) $(n=15)$ received Casley-Smith MLD technique and bandage in the following steps. The affected limb and trunk were per skin and the therapist applied very soft pressure on patient's skin and subdermal tissues of the affected extremity. The therapist applied pressure for $40 \mathrm{~min}$, including 30 min for the trunk and 10 min for the affected limb starting with the distal area and trying to move the lymph into the non-evacuated parts, while proximal areas are avoided.

Bandaging is applied at the end of each session. Bandages were wrapped using a high pressure gradient at the most distal end of the limb which gradually decreases proximally. A layer of cotton (Velband) is applied firstly and then followed by protection at the points of support. A bandage of low elasticity (Comprilan) of various widths (using $6 \mathrm{~cm}$ for the toes and $8 \mathrm{~cm}$ for the remaining of the extremity) was used.

\section{Cellulite assessment}

By using the Cellulite Severity Scale, the researchers made group comparisons at the initial visit and after 8 weeks of treatment.
The Cellulite Severity Scale has the best reliability and internal consistency when utilized to assess posterior thighs and buttocks cellulite [12].

CSS scale distinguishes 5 key clinical morphologic features of cellulite: (1) quantity of visible depressions, (2) depressions depth, (3) morphologic changes in skin surface, (4) degree of skin laxity or sagging, and (5) and Nürnberger and Müller classification scale. The seriousness of every item ranges from 0 to 3 , where 0 represents the absence of cellulite and 3 represents the severe degree of cellulite. This made the total count of scores ranging from 0 to 15 . According to the total numeric score, cellulite is classified as mild (1-5), moderate (6-10), or severe (11-15). All measurements of each patient were taken before, after 8 weeks and at the end of treatment.

When using the Nürnberger and Müller classification scale for cellulite, patients should be examined in the erect position with the gluteus muscles in a relaxed position. If the patient does not have obvious depressions, the pinch test must be applied (by pinching the skin between the thumb and the index finger). It includes 4 grades: grade (zero): there are no changes in the skin surface, grade (I): the skin is smooth in the standing or lying position, but the changes in the skin can be seen by pinching the skin, grade (II): the orange skin or mattress appearance is obvious in the standing position, without pinching and grade (III): the same finding as in grade II with raised areas and nodules $[13,14]$.

\section{Statistical analysis}

Descriptive statistics with unpaired t-test was used for comparing between subject characteristics of the groups. Cellulite Severity Scale scores were compared between groups by Mann-Whitney $U$ test and between preand post-treatment within groups by Wilcoxon Signed Ranks. The level of significance for all statistical tests was set at $p<0.05$. All statistical analyses were performed by the statistical package for social studies (SPSS) version 22 (IBM SPSS, Chicago, IL, USA).

\section{Results}

\section{Subject characteristics}

Table 1 shows the characteristics of subjects in group $A$ and $B$. There was no significant difference between group $A$ and $B$ in the mean age $(p>0.05)$.

\section{Effect of treatment on Cellulite Severity Scale score}

The distribution of Cellulite Severity Scale before the treatment showed that $100 \%$ of the subjects in both groups had severe grades (11-15). After the treatment, $13(86.7 \%)$ subjects in group A and $12(80 \%)$ subjects in group B had mild grades (1-5); while 2 (13.3\%) subjects in group $A$ and $3(20 \%)$ subjects in group $B$ had moderate grades (6-10) (Table 2). 
Table 1. Comparison of subject characteristics between group $A$ and $B$

\begin{tabular}{|c|c|c|c|c|c|}
\hline \multirow[t]{2}{*}{ Variable } & \multicolumn{2}{|c|}{$\bar{x} \pm \mathrm{SD}$} & \multirow[t]{2}{*}{$M D$} & \multirow[t]{2}{*}{$t$-value } & \multirow[t]{2}{*}{$P$-value } \\
\hline & Group A & Group B & & & \\
\hline Age [years] & $40.86 \pm 2.79$ & $39.73 \pm 3.23$ & 1.13 & 1.02 & 0.31 \\
\hline Maximum-minimum & $45-36$ & $45-35$ & & & \\
\hline
\end{tabular}

$\bar{x}$-Mean, SD - standard deviation, MD - mean difference, $p$-value - probability value.

Table 2. Comparison of subject characteristics between group A and B

\begin{tabular}{lcc}
\hline $\begin{array}{l}\text { Cellulite Severity } \\
\text { Scale score }\end{array}$ & $\begin{array}{c}\text { Group A } \\
\text { Frequency }\end{array}$ & $\begin{array}{c}\text { Group B } \\
\text { Frequency }\end{array}$ \\
\hline Pre treatment: & $0(0 \%)$ & $0(0 \%)$ \\
\hline Mild (1-5) & $0(0 \%)$ & $0(0 \%)$ \\
\hline Moderate (6-10) & $15(100 \%)$ & $15(100 \%)$ \\
\hline Severe (11-15) & $13(86.7 \%)$ & $12(80 \%)$ \\
\hline Post treatment: & $2(13.3 \%)$ & $3(20 \%)$ \\
\hline Mild (1-5) & $0(0 \%)$ & $0(0 \%)$ \\
\hline Moderate (6-10) &
\end{tabular}

There was a significant decrease in median values of Cellulite Severity Scale score after treatment in group $\mathrm{A}$ and $\mathrm{B}$ compared with that before the treatment $(p>$ 0.001). The median value of Cellulite Severity Scale score decreased from 12 (severe) to 4 (mild) in both groups (Table 3).

There was no significant difference in Cellulite Severity Scale score between both groups before the treatment $(p>0.05)$. Comparison between group A and B after the treatment revealed a non-significant difference in Cellulite Severity Scale score between the groups $(p>0.05)$ (Table 3, Figure 1).

\section{Discussion}

Cellulite is regularly utilized to characterize a complex structural issue within the skin with multiple causes. Cellulite occurrence is a common process and not pathology, and occurs in approximately $85 \%$ in grown-up women. The appearance of cellulite has a negative impact on the quality of life in women and they suffer from dimpling skin like orange peel [15].

Cellulite pathophysiology is not clearly known, but it is common besides a disturbed connective tissue structure and a changed extracellular matrix within the dermal layers. Obesity also plays an additional detrimental role on cellulite-prone skin zones such as the buttocks and thighs. Indeed, in spite of the fact that cellulite arrangement is aggravated in combination with obesity,
Table 3. Median values of Cellulite Severity Scale score for group A and B pre- and post-treatment

\begin{tabular}{lcccc}
\hline $\begin{array}{l}\text { Cellulite Severity } \\
\text { Scale score }\end{array}$ & $\begin{array}{c}\text { Group A } \\
\text { Median } \\
(\text { IQR })\end{array}$ & $\begin{array}{c}\text { Group B } \\
\text { Median } \\
(\text { IQR })\end{array}$ & $U$-value & $P$-value \\
\hline Pre treatment & $12(13.11)$ & $12(13.11)$ & 108.5 & 0.86 \\
\hline Post treatment & $4(5.3)$ & $4(5.3)$ & 110 & 0.91 \\
\hline Z-value & 3.47 & 3.44 & & \\
\hline & $p=0.001$ & $p=0.001$ & & \\
\hline
\end{tabular}

IQR - inter quartile range, U-value - Mann-Whitney test value, Z-value - Wilcoxon signed ranks test value, $p$-value - level of significance.

it is overwhelmingly subordinate on the perpendicular alignment and conditions of dermal septae with respect to the skin surface. These dermal septae are more radially situated and thinner in women with cellulite compared to unaffected men and women [16].

It is known that the enhancements of skin quality and dermal flexibility are two vital restorative points

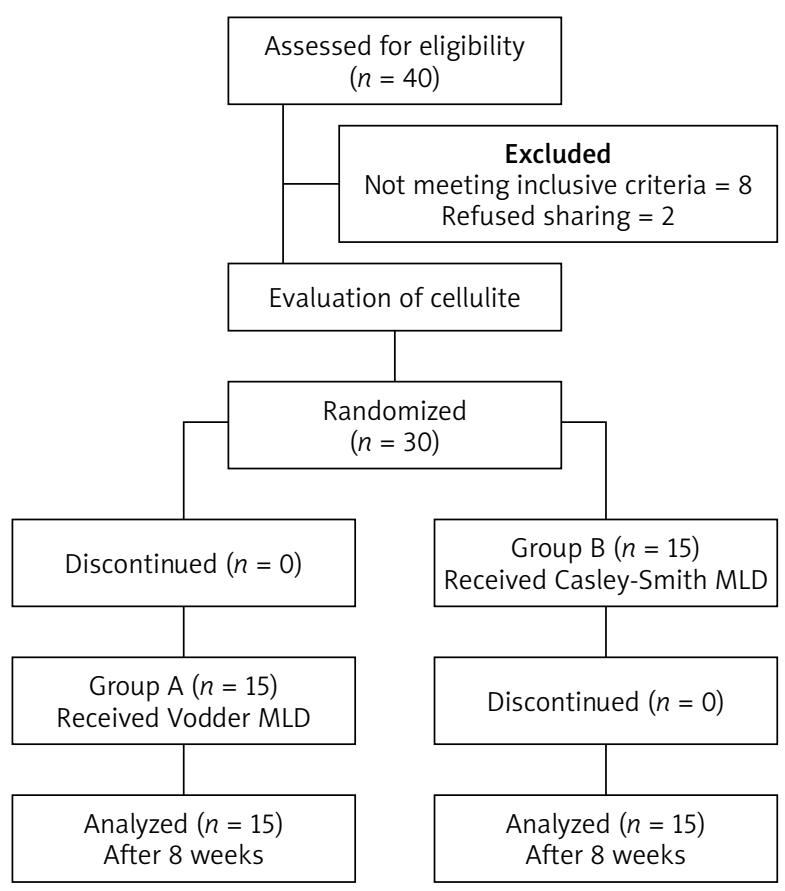

Figure 1. Participants' flowchart 
in cellulite enhancements. Unexpectedly, it is observed that, notwithstanding of age, the newness of cellulite is compared to decay of the skin layer, an increment of the length of the dermis-hypodermis interface, and decay of dermal thickness and mechanical properties of skin versatility and compliance that are adversely influenced by the severity of cellulite [17].

It is noted that women that are cellulite free had a better skin condition (more tightness, less laxity, compliance and distortion ability) in the upper back portion of the thigh. On the other hand, women with cellulite have more loosening and shortcoming of the skin and soft tissue that is amplified into the superficial layers of fascia [18].

The current study was a randomized control trial to compare between Vodder MLD technique and CasleySmith MLD technique for cellulite after liposuction. The participants were randomly assigned to one of two treatment groups: Group (A) received Vodder MLD technique and bandage while Group (B) received Casley-Smith MLD technique and bandage. The researchers made group comparisons at the initial visit and after 8 weeks. The duration of the intervention was 8 weeks per participant, and each participant received 3 sessions per week.

The outcomes of this research showed a significant decrease in median values of Cellulite Severity Scale score before treatment in group $A$ and $B$ comparing to that after treatment. The median value of Cellulite Severity Scale score decreased from 12 (severe) to 4 (mild) in the two groups.

There was no significant difference in Cellulite Severity Scale score between both groups before treatment. Comparison between group A and B after treatment showed a non-significant difference in Cellulite Severity Scale score between the groups.

A study was applied to assess contour reductions in the clinical management of cellulite. The therapeutic records of 150 patients treated for cellulite were reviewed. Treatment of mechanical and Vodder manual lymph drainage, with cervical stimulation was performed for 1 daily for 10 days. A critical decrease in contour was noticed after the therapeutic management of cellulite showing an enhancement in the aesthetics [19].

Another study was applied for assessment of $10 \mathrm{pa}$ tients with cellulite grade IV. Before starting the treatment, contour measurement was made at intervals of $5 \mathrm{~cm}$ along the thigh, at the level, and 5,10 , and $15 \mathrm{~cm}$ above the gluteal fold, at the level, and $5 \mathrm{~cm}$ above the umbilicus. The patients were provided with a 4-hour daily therapeutic session that comprised of Vodder manual and mechanical lymph drainage and stimulation of the cervical lymph nodes. At the measurement points, the major decrease ranged between 4.0 and $5.7 \mathrm{~cm}$, although a decrease greater than $10 \mathrm{~cm}$ in contour was accomplished in a few patients. This study found that lymphatic stimulation is effective in cellulite treatment [20].
Another study was applied to assess the short-term outcomes of pressure garment with Manual Lymphatic Drainage on the lymphatic system by Near-Infrared Imaging (NIR) explored. Nine healthy volunteers underwent NIR fluoroscopy to quantify lymphatic behaviour before and after a 15-minute period of Casley-Smith manual lymph drainage followed by compression garment (CG) therapy for a 10-minute period. Images were made at the forearm and elbow after each intervention. The lymph parameters were analyzed to assess the effects of the interventions compared with basal values. Baseline (BL) parameters of lymph function revealed high variability in the number, size, and speed of packets between individuals. Both mediations appeared to be a statistically critical advancement $(p<0.05)$ in displacement and speed at the forearm compared with standard. It was concluded that lymphatic action expanded significantly after MLD [21].

In addition, a study was applied to assess the effect of MLD associated with ultrasound for treating women with cellulite; the study was applied on 10 women with cellulite. MLD was connected for 60 min and the US was utilized in the buttocks and upper thighs with a frequency of $3 \mathrm{MHz}$ with an intensity of $0.6 \mathrm{~W} / \mathrm{cm}^{2}$, continuous mode and a duration of $12 \mathrm{~min}$. A significant improvement in the grade of cellulite at the buttocks and in the patient's satisfaction with cellulite compared before and after the treatment was found. The study concluded that manual lymphatic drainage is viable in treating cellulite and ultrasound improves its effect leading to high patient satisfaction [22].

Another study applied to examine the safety and effectiveness of manual lymphatic drainage for cellulite reduction and included twenty women aged from twenty to 40 years. Fourteen sessions of manual lymphatic drainage were conducted, one session per week, on the gluteal region and thighs. A significant decrease, on average of $0.3 \pm 0.8 \mathrm{~cm}$, in the hip contour was noted, with no difference in thighs contour $(p>0.05)$. The study concluded that manual lymphatic drainage is secure but still not viable as a single method for cellulite treatment [23].

In our present research, we recognize the various limitations, such as the lack of a placebo (control) arm, the lack of long-term follow-up and the limited number of participating patients.

\section{Conclusions}

Both Vodder MLD technique and Casley-Smith MLD technique have a positive effect in reducing cellulite after thigh liposuction but there was no significant difference between Vodder MLD technique and Casley-Smith MLD technique for treatment of cellulite after thigh liposuction, hence any technique of MLD is recommended to achieve better improvement. 


\section{Acknowledgments}

The authors would like to thank the participating patients for their enthusiasm and patience.

\section{Conflict of interest}

The authors declare no conflict of interest.

\section{References}

1. Bellini E, Grieco MP, Raposio E. A journey through liposuction and liposculture. Ann Med Surg 2017; 24: 53-60.

2. Tokarska K, Tokarski S, Woźniacka A, et al. Cellulite: a cosmetic or systemic issue? Contemporary views on the etiopathogenesis of cellulite. Adv Dermatol Allergol 2018; 35: $442-6$.

3. de Godoy JM, de Godoy MD. Treatment of cellulite based on the hypothesis of a novel physiopathology. Clin Cosmet Investig Dermatol 2011; 4: 55-9.

4. Kravitz L, Achenbach NJ. Cellulite: a review of its anatomy, physiology and treatment. IDEA Fit J 2010; 7: 36-43.

5. Hexsel DM, Dal'Forno T, Hexsel CL. A validated photonumeric cellulite severity scale. J Eur Acad Dermatol Venereol 2009; 23: 523-8.

6. Khan MH, Victor F, Rao B, Sadick NS. Treatment of cellulite: part II. Advances and controversies. J Am Acad Dermatol 2010; 62: 373-84.

7. De Godoy JM, De Godoy MD. Physiopathological hypothesis of cellulite. Open Cardiovasc Med J 2009; 3: 96-7.

8. Ezzo J, Manheimer E, McNeely ML, et al. Manual lymphatic drainage for lymphedema following breast cancer treatment. Cochrane Database Syst Rev 2015; 5: CD003475.

9. Flórez-García MT, Valverde-Carrillo MD. Eficacia del tratamiento conservador no farmacológico del linfedema postmastectomía. Rehabilitación 2007; 41: 126-34.

10. Zuther JE, Norton S, Armer JM. Lymphedema management: the comprehensive guide for practitioners. Thieme, New York 2009.

11. Casley-Smith JR, Boris M, Weindorf S, Lasinski B. Treatment for lymphedema of the arm-The Casley-Smith method: a noninvasive method produces continued reduction. Cancer 1998; 83: 2843-60.

12. De La Casa Almeida M, Suarez Serrano C, Jiménez Rejano JJ, et al. Intra-and inter-observer reliability of the application of the cellulite severity scale to a Spanish female population. J Eur Acad Dermatol Venereol 2013; 27: 694-8.

13. Nürnberger F, Müller G. So-called cellulite: an invented disease. J Dermatol Surg Oncol 1978; 4: 221-9.

14. Hexsel D. Cellulite. In: Women's dermatology. Parthenon Publishing Group 2001; 586-95.

15. Khan MH, Victor F, Rao B, Sadick NS. Treatment of cellulite: part I. Pathophysiology. J Am Acad Dermatol 2010; 62: 361-70.

16. Mirrashed F, Sharp JC, Krause V, et al. Pilot study of dermal and subcutaneous fat structures by MRI in individuals who differ in gender, BMI, and cellulite grading. Skin Res Technol 2004; 10: 161-8.

17. Ortonne JP, Zartarian M, Verschoore M, et al. Cellulite and skin ageing: is there any interaction? J Eur Acad Dermatol Venereol 2008; 22: 827-34.

18. Dobke MK, DiBernardo B, Thompson RC, Usal H. Assessment of biomechanical skin properties: is cellulitic skin different? Aesthet Surg J 2002; 22: 260-6.
19. de Godoy JM, de Godoy AC, Godoy MD. Considering the hypothesis of the pathophysiology of cellulite in its treatment. Dermatol Rep 2017; 9: 7352

20. de Godoy JM, Groggia MY, Ferro Laks L, Guerreiro de Godoy MD. Intensive treatment of cellulite based on physiopathological principles. Dermatol Res Pract 2012; 2012: 834280.

21. Lopera C, Worsley PR, Bader DL, Fenlon D. Investigating the short-term effects of manual lymphatic drainage and compression garment therapies on lymphatic function using near-infrared imaging. Lymphat Res Biol 2017; 15: 235-40.

22. de Almeida AF, Brandão DS, Silva JC, et al. Evaluation of manual lymphatic drainage and ultrasound in the cellulite. Rev Aten Saúde 2011; 9(28).

23. Schonvvetter B, Soares JL, Bagatin E. Longitudinal evaluation of manual lymphatic drainage for the treatment of gynoid lipodystrophy. An Bras Dermatol 2014; 89: 712-8. 